\title{
Elongational crystallization of isotactic polypropylene forms nano-oriented crystals with ultra-high performance
}

\author{
Kiyoka N Okada ${ }^{1,2}$, Jun-ichiro Washiyama ${ }^{3}$, Kaori Watanabe ${ }^{2}$, Sono Sasaki ${ }^{4}$, Hiroyasu Masunaga ${ }^{4}$ and \\ Masamichi Hikosaka ${ }^{1,2}$
}

\begin{abstract}
Although the large 'melt elongation' of polymers should significantly affect crystallization that controls the structure and properties of solids, it has been difficult due to technical reasons. In this paper, we succeeded in bulky elongational crystallization of isotactic polypropylene (iPP) by compressing the supercooled melt. The crystallization behavior and structure were observed by means of optical microscope, transmission electron microscopy (TEM) and X-ray scattering. When the elongational strain rate $(\dot{\varepsilon})$ surpassed a critical value $\left(\dot{\varepsilon}^{*}=2 \times 10^{2} \mathrm{~s}^{-1}\right)$, the crystallization behavior, structure and physical properties underwent a discontinuous change. The crystallization rate increased by a factor as large as $10^{6}$, the crystallite lateral size decreased by a factor of $10^{-3}$ to $20-30 \mathrm{~nm}$ and the morphology changed from well-known spherulites to a novel morphology of 'nano-oriented crystals (NOCs)'. These NOCs showed a high crystallinity (nearly equal to unity). The above results indicate that the polymer melt changed from isotropic to 'oriented melt' which accelerated nucleation and growth rates and induced NOC formation. NOCs showed ultra-high performance such as high tensile strength at break ( $\cong 2.1 \times 10^{2} \mathrm{MPa}$ ), comparable to that of metals, and a high thermal resistance $\left(\cong 176^{\circ} \mathrm{C}\right)$. NOCs will be useful in a wide variety of applications and will contribute to efforts to construct a sustainable society by enabling the development of lightweight, recyclable materials.
\end{abstract}

Polymer Journal (2010) 42, 464-473; doi:10.1038/pj.2010.35

Keywords: crystallization; elongation; isotactic polypropylene; nano; SAXS; WAXS

\section{INTRODUCTION}

String- or chain-like molecules are major constituents of natural materials, as they have the remarkable characteristic of a high ability to self-organize. Because these molecules can form complex structures, they perform multiple functions and can carry information. The functions of these molecules and the information they carry have evolved over more than three billion years. Chain-like materials, called polymers, are now used in a variety of applications and have properties similar to those of the natural string-like molecules discussed above. Therefore, it is important for us to understand and control the behaviors of the structure formation of polymer.

The molecular chain of a polymer in a melt or solution significantly changes its shape if an external field, such as an elongation, changes from weak to strong one, ${ }^{1}$ which should significantly affect the crystallization behavior and properties of polymer solids. Therefore, the mechanism of structure formation and the expression of function of polymers under strong elongational fields are interesting problems in both scientific and industrial fields. However, these problems have not yet been studied or solved because it has so far been impossible to achieve such a strong melt elongation because of technical difficulties. ${ }^{2}$

Crystallizations under a quiescent field and/or under a weak external field have been well studied for half a century. ${ }^{3}$ Conventional polymer solids have been crystallized under quiescent or weak external fields. Melted polymer chains spontaneously take the shape of random coils, referred to as Gaussian chains, because of entropy, and they easily become entangled with each other in the quiescent field. This type of melt is referred to as an isotropic melt. In the case of a weak external field, an isotropic melt has the greatest volume. ${ }^{4}$ Polymer chains must be ordered from the isotropic melt during crystallization. ${ }^{5}$ However, this ordering is difficult because it is not easy for the entangled chains to slide along their chain axes and disentangle into an ordered parallel arrangement; therefore, amorphous areas remain in the solid. Thus, conventional polymer solids are composed of amorphous and crystal parts, that is, the crystallinity $\left(\chi_{\mathrm{c}}\right)$ is much less

${ }^{1}$ Graduate School of Integrated Arts and Sciences, Hiroshima University, Kagamiyama, Higashi-hiroshima city, Hiroshima, Japan; ${ }^{2}$ Innovation Plaza Hiroshima, Japan Science and Technology Agency, Kagamiyama, Higashi-hiroshima city, Hiroshima, Japan; ${ }^{3}$ Kawasaki Development Center, SunAllomer Ltd, Yako, Kawasaki-ku, Kawasaki city, Kanagawa, Japan and ${ }^{4}$ SPring-8, Japan Synchrotron Radiation Research Institute, Kouto, Sayo-cho, Sayo-gun, Hyogo, Japan

Correspondence: Professor M Hikosaka, Graduate School of Integrated Arts and Sciences, Japan/Hiroshima University, Kagamiyama 1-7-1, Higashi-hiroshima city, Hiroshima 739-8521, Japan.

E-mail: hikosaka@hiroshima-u.ac.jp

Received 16 December 2009; revised 16 March 2010; accepted 18 March 2010 
a
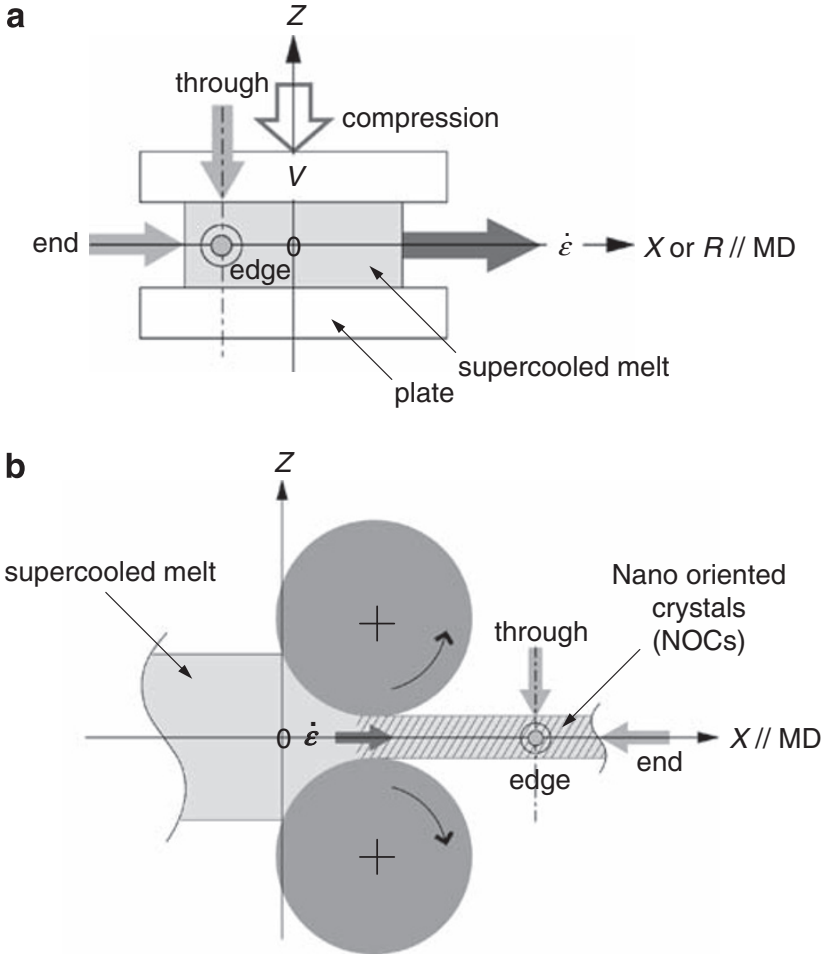

Figure 1 Schematic illustration of a cross-sectional view of crystallization apparatuses shows the principle of elongational crystallization by the compression method. The $Z$ axis is along the direction of compression. The $X$ - or $R$-axis is parallel to the machine direction (MD). (a) Compression-type apparatus. (b) Roll-type apparatus.

than unity. ${ }^{6}$ Such polymer solids perform poorly for properties such as tensile strength, as the amorphous portion takes on the structure of the frozen melt.

In this paper, we succeeded in realizing a strong melt elongation for the first time by developing a new 'compression method'. Figure 1a shows the principle of melt elongation by the compression method. We compressed the supercooled melt by sandwiching it between two plates, thus stretching the melt along a direction perpendicular to the compressed direction. Figure $1 \mathrm{~b}$ shows a roll-type apparatus that can also apply a strong compression to the sample as described later. In this way, we were able to impart effective elongation to the melt with strong and fast compression, resulting in a significant change in the melt structure from the isotropic melt to the oriented melt and in the crystallization behaviors and properties of crystallized polymer solids.

\section{Purpose}

The purposes of this study are as follows: (1) to show that nanooriented crystals (NOCs) are formed by elongational crystallization under a strong elongational field; (2) to clarify the structure and nucleation mechanism of NOCs; and (3) to show that NOC demonstrate ultra-high performance in physical properties because of their fine structure.

\section{Theory}

We developed one-dimensional (1D) and two-dimensional (2D) compression methods. In the case of $1 \mathrm{D}$ compression, the width of the sample is kept constant during compression. In the case of $2 \mathrm{D}$ compression, a rod sample is compressed into a disk.

The degree of melt elongation can be quantitatively expressed by $\dot{\varepsilon}$, which is approximately formulated as follows: We use a relative coordinate system and take the center of the sample as the origin (Figures $1 \mathrm{a}$ and $\mathrm{b}$ ), where $R$ is the radial direction. $X$ - or $R$-axes corresponds to the direction of elongation and is called machine direction (MD). The initial size of a supercooled melted sample is denoted by $\left(2 X_{0}, 2 Y_{0}, 2 Z_{0}\right)$ or $\left(2 R_{0}, 2 Z_{0}\right)$ for $1 \mathrm{D}$ or $2 \mathrm{D}$ compression, respectively. The size of the sample at the time $(t)$ of compression is denoted by $\left(2 X, 2 Y_{0}, 2 Z\right)$ or $(2 R, 2 Z)$ for $1 \mathrm{D}$ or $2 \mathrm{D}$ compression, respectively. $\dot{\varepsilon}$ along the MD is defined by ${ }^{7}$

$$
\begin{aligned}
\dot{\varepsilon}=|\dot{\varepsilon}| & \equiv \frac{1}{X} \cdot \frac{\mathrm{d} X}{\mathrm{~d} t} \quad \text { for } 1 \mathrm{D} \text { compression } \\
& \equiv \frac{1}{R} \cdot \frac{\mathrm{d} R}{\mathrm{~d} t} \quad \text { for } 2 \mathrm{D} \text { compression }
\end{aligned}
$$

Compression velocity $(v)$ is defined by

$$
v=|\boldsymbol{v}| \equiv-\frac{\mathrm{d}(2 \mathrm{Z})}{\mathrm{d} t}
$$

From the mass conservation law, we have

$$
X_{0} Y_{0} Z_{0}=X Y_{0} Z \text { for } 1 \mathrm{D} \text { compression }
$$

or

$$
\pi R_{0}^{2}\left(2 Z_{0}\right)=\pi R^{2}(2 Z) \text { for } 2 \mathrm{D} \text { compression }
$$

Combination of equations 1,2 and 3 gives

$$
\begin{aligned}
& \dot{\varepsilon}=\frac{v}{2 Z} \quad \text { for } 1 \mathrm{D} \text { compression along the } X \text {-axis } \\
& =\frac{v}{4 Z} \quad \text { for } 2 \mathrm{D} \text { compression along the } R \text {-axis }
\end{aligned}
$$

In this study, we approximated $v$ by

$$
v \cong \frac{2\left(Z_{0}-Z\right)}{\Delta t}
$$

where $\Delta t$ is the compression time. Although $\dot{\varepsilon}$ depends on the thickness of the sample, we simply approximated $\dot{\varepsilon}$ by the average value. We can change $\dot{\varepsilon}$ by changing $v$ and the sample thickness. $\dot{\varepsilon}$ for rolling-type compression could also be given by equation $4 \mathrm{a}$ in principle.

\section{EXPERIMENTAL PROCEDURE}

\section{Sample}

We used $\operatorname{iPP}\left(M_{\mathrm{w}}=34 \times 10^{4}, M_{\mathrm{w}} / M_{\mathrm{n}}=30[\mathrm{mmmm}]=98 \%\right)$ as a model polymer. The equilibrium melting temperature $\left(T_{\mathrm{m}}{ }^{0}\right)$ of $\alpha_{2}^{\prime}$ form under a quiescent field was $187^{\circ} \mathrm{C} .8,9$

\section{Crystallization under elongational field}

For 1D compression-type crystallization, we developed a roll-type crystallization apparatus (Figure 1b). The sample was melted within an extruder at a temperature above $T_{\mathrm{m}}{ }^{0}$ (the maximum temperature $T_{\max }=240^{\circ} \mathrm{C}$ ), cooled to a crystallization temperature $\left(T_{\mathrm{c}}=150^{\circ} \mathrm{C}\right)$ and then rolled to elongate the supercooled melt into a sheet between two rotating rolls with diameters of $200 \mathrm{~mm}$. The temperature of the rolls was also controlled at $T_{\mathrm{c}}=150{ }^{\circ} \mathrm{C}$. The roll gap was changed from 0.1 to $1 \mathrm{~mm}$. This rolling of the 'supercooled melt' at $T_{\mathrm{c}}$ is the first demonstration of roll-type processing. In conventional roll- or cast-type processing, a melted sample maintained at a much higher temperature than $T_{\mathrm{c}}$ should be guided into rolls maintained at a much lower temperature than $T_{\mathrm{c}}$. The range of $\dot{\varepsilon}$ was changed from 0 to $10^{3} \mathrm{~s}^{-1}$ by changing the rotating velocity of the rolls. The sample was rolled with a constant rolling velocity, which means that the sample experienced uniaxial elongation. The average $\dot{\varepsilon}$ was evaluated using equation $4 \mathrm{a}$.

In the case of $2 \mathrm{D}$ compression (Figure 1a), a rod sample was melted at $T_{\max }=203{ }^{\circ} \mathrm{C}$ (above $T_{\mathrm{m}}{ }^{0}$ ) for $3 \mathrm{~min}$, cooled to $T_{\mathrm{c}}=150^{\circ} \mathrm{C}$ and then compressed into a disk shape between two plates. $\dot{\varepsilon}$ ranged from 0 to $3 \times 10^{3} \mathrm{~s}^{-1}$. 


\section{Instruments}

The crystallization behavior and optical morphology of the sample were observed using a polarizing optical microscope (POM) (BX51N-33P-OC, Olympus Corporation, Tokyo, Japan) and a Digital charge-coupled device camera system (QICAM, QImaging, Tokyo, Japan) or a high-speed camera (Memrecam fx K5, NAC Image Technology Inc., Tokyo, Japan). Although the frame rate of the high-speed camera was $10^{5} \mathrm{fps}$, the actual frame rate was $10^{3}$ fps because of the limit of brightness of the optical light. Therefore, the time resolution of this system was $1 \mathrm{~ms}$. Crystallization was directly observed using a compression apparatus shown in Figure 1 by OM along the 'through direction'. Here, we observed the sample along three directions, that is, through, edge and end (Figure 1). The through direction is parallel to the direction of compression, edge direction is perpendicular to through and $\mathrm{MD}$, and the end direction is parallel to the MD.

Samples were observed by transmission electron microscopy (TEM) using a JEOL JEM1200EX (JEOL Ltd, Tokyo, Japan) with an acceleration voltage of $120 \mathrm{kV}$. Samples were prepared for TEM as follows: Specimens were embedded in epoxy resin and trimmed using Reichert Ultracut S (Reichert-Nissei, Tokyo, Japan) equipped with glass and trimming knives. They were then stained by a $0.5 \mathrm{wt} \%$ solution of $\mathrm{RuO}_{4}$ for $24 \mathrm{~h}$. Ultrathin sections about $70 \mathrm{~nm}$ thick of three views of the specimen were obtained using a microtome equipped with a diamond knife at room temperature. We observed the sample along the through, edge and end directions.

Small/wide-angle X-ray scattering (SAXS/WAXS) observations were carried out using synchrotron radiation at beam line BL40B2 of SPring-8 at the Japan Synchrotron Radiation Research Institute (JASRI) in Harima City. The SAXS and WAXS intensities of the samples were obtained by correcting the background intensity. The incident X-ray beam was exposed to the sample along the through, edge and end directions.

\section{Orientation function}

Orientation function $\left(f_{c}\right)$ is a well-known order parameter that expresses the degree of chain orientation. ${ }^{10} f_{\mathrm{c}}$ was measured using the half-width of 130 reflection.

\section{Crystallinity $\chi_{\mathrm{c}}$}

$\chi_{\mathrm{c}}$ was obtained by determining sample density $(\rho)$ by measuring the mass $(M)$ and volume $(V)$ of a sample. As $V$ was precisely measured by means of a gas densimeter (micromeritics gas pycnometer, AccuPyc 1340, Shimadzu, Kyoto, Japan) with a precision of $0.05 \%, \chi_{c}$ was measured within a $1 \%$ error.

\section{Physical properties}

Tensile properties were tested by applying a procedure compliant with JIS K7161 (ISO527-1) using a tension tester (Shimadzu, accurate universal testing machine, Autograph AG-1k NIS). The tensile properties were observed at room temperature with a strain rate of $0.2 \mathrm{~s}^{-1}$.

Toughness was semiquantitatively evaluated by folding the material up to 100 times along the MD or transverse direction (TD).

A thermal resistance test was carried out using a hot stage (L-600 A, Linkham Scientific Instruments Ltd, Tadworth, UK) and POM and recorded by a charge-coupled device camera system. Square cut samples were placed in the hot stage and heated from room temperature to $240^{\circ} \mathrm{C}$ at a heating rate of $1 \mathrm{~K} \mathrm{~min}^{-1}$. We observed the lateral length of the sample along the MD and TD, designated as $l_{\mathrm{MD}}$ and $l_{\mathrm{TD}}$, respectively. Heatproof temperature $\left(T_{\mathrm{h}}\right)$ was defined as the temperature at which $l / l_{0}$ shrank or expanded more than $3 \%$ with an increase in $T$.

Transparency was evaluated by so-called 'haze'. Haze was obtained from optical density using a calibration curve between optical density and the haze. The calibration curve was constructed from 20 referential iPP sheet samples. Haze was obtained by a method compliant with JIS K7136 (ISO14782). The haze in this study was defined for sheets of $0.3 \mathrm{~mm}$ thickness. The optical density was obtained by analyzing the optical image observed by POM and the charge-coupled device camera system mentioned above using imaging software (Image-Pro PLUS, Media Cybernetics Inc., Bethesda, MD, USA).

\section{RESULTS}

\section{$\dot{\varepsilon}$ dependence of morphology observed by OM}

Figure 2 shows typical polarizing optical micrographs of samples after the completion of crystallization at $150^{\circ} \mathrm{C}$ by $1 \mathrm{D}$ (Figures $2 \mathrm{~b}$ and $\mathrm{d}$ ), 2D (Figures 2a, c and e) compressions.

Large spherulites were crystallized at quiescent field $\left(\dot{\varepsilon}=0 \mathrm{~s}^{-1}\right)$ (Figure 2a). The mean diameter $(d)$ of the spherulites was $d \geqslant 1.5 \times 10^{2} \mu \mathrm{m}$. When $\dot{\varepsilon}$ increased, $d$ became as small as several tens of $\mu \mathrm{m}$ (Figures $2 \mathrm{~b}$ and $\mathrm{c}$ ).

When $\dot{\varepsilon}$ became larger than $2 \times 10^{2} \mathrm{~s}^{-1}$, the morphology changed from spherulites to a new morphology (Figures $2 \mathrm{~d}$ and e). The crystal size became too fine to be detected by POM, which suggested that NOCs were generated. Detailed evidence of the formation of NOCs will be presented later. The characteristic colored pattern (corresponding to retardation ${ }^{11}$ ) indicates that molecular chains are

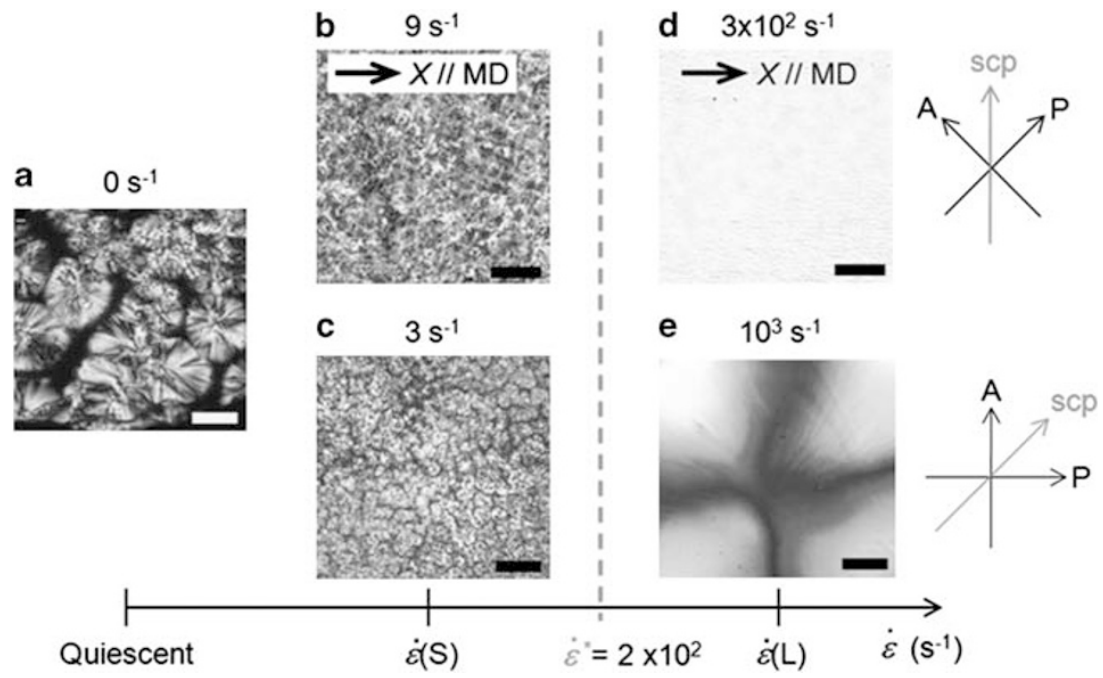

Figure 2 Typical polarizing optical morphology of iPP samples crystallized at $150{ }^{\circ} \mathrm{C}$ (a), crystallized in a quiescent field, $\dot{\varepsilon}=0 \mathrm{~s}^{-1}$. The scale bar is $200 \mu \mathrm{m}$, and the sample thickness is $70 \mu \mathrm{m}$. (b) $\dot{\varepsilon}(\mathrm{S})=9 \mathrm{~s}^{-1}$. Sample thickness is $0.27 \mathrm{~mm}$. (c) $\dot{\varepsilon}(\mathrm{S})=3 \mathrm{~s}^{-1}$. Sample thickness is $90 \mu \mathrm{m}$. (d) $\dot{\varepsilon}(\mathrm{L})=3 \times 10^{2} \mathrm{~s}^{-1}$. Sample thickness is $0.25 \mathrm{~mm}$. (e) $\dot{\varepsilon}(\mathrm{L})=10^{3} \mathrm{~s}^{-1}$. Sample thickness is $10 \mu \mathrm{m}$. (b, d) Crystallized by 1D compression. Scale bar is $50 \mu \mathrm{m}$. (c, e) Crystallized by $2 \mathrm{D}$ compression. Scale bar is $200 \mu \mathrm{m}$. Arrows on the right side indicate the directions of polarizer (P), analyzer (A) and sensitive-color plate (scp). 


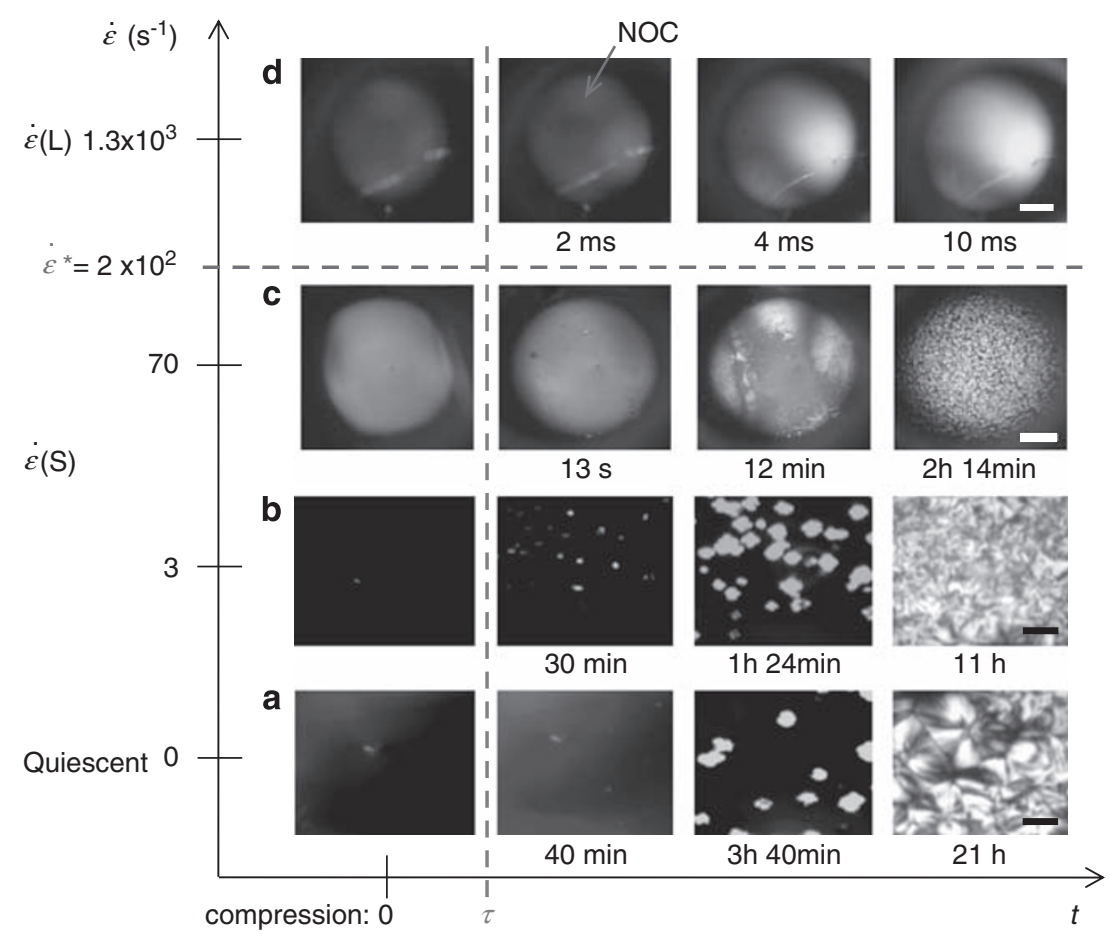

Figure 3 Typical $\dot{\varepsilon}$ dependence of crystallization behavior directly observed by optical microscopy on samples elongationally crystallized by $2 \mathrm{D}$ compression. $t$ is crystallization time. At $t=0$, a sample had just been compressed. Samples were crystallized at (a) quiescent field, $\dot{\varepsilon}=0 \mathrm{~s}^{-1}$, (b) $\dot{\varepsilon}(\mathrm{S})=3 \mathrm{~s}^{-1}$, (c) $\dot{\varepsilon}(\mathrm{S})=70 \mathrm{~s}^{-1}$ and (d) $\dot{\varepsilon}(\mathrm{L})=1.3 \times 10^{3} \mathrm{~s}^{-1}$. (a, b) Scale bar is $100 \mu \mathrm{m}$. (c, d) Scale bar is $500 \mu \mathrm{m}$.

oriented along the $X$ - and $R$-axes for $1 \mathrm{D}$ and $2 \mathrm{D}$ compressions, respectively. Hence, the chains are oriented along the MD for both $1 \mathrm{D}$ and 2D compressions. Therefore, we found that there is a 'critical' point in $\dot{\varepsilon}$, which we name the critical elongational strain rate $\left(\dot{\varepsilon}^{\star}\right)$ and later evaluate as $\dot{\varepsilon}^{\star}=2 \times 10^{2} \mathrm{~s}^{-1}$ using an X-ray method.

Hereafter, we will classify $\dot{\varepsilon}$ into $\dot{\varepsilon}(\mathrm{S})$ and $\dot{\varepsilon}(\mathrm{L})$, where $\dot{\varepsilon}(\mathrm{S})<\dot{\varepsilon}^{*}$ and $\dot{\varepsilon}(\mathrm{L}) \geqslant \dot{\varepsilon}^{\star}$, respectively. For the sake of simplification, samples crystallized at $\dot{\varepsilon}(\mathrm{S})$ and $\dot{\varepsilon}(\mathrm{L})$ will be named $\dot{\varepsilon}(\mathrm{S})$ and $\dot{\varepsilon}(\mathrm{L})$, respectively.

Although shear should exist at the interface between the sample and plates, no different morphologies such as 'skin' were detected at the interface of the sample by means of OM, X-ray scattering or TEM, as shown later. This observation indicates that shear rate is much less effective than $\dot{\varepsilon}$ in inducing crystallization above $\dot{\varepsilon}^{\star}$. This result agrees well with Yamamoto's theoretical prediction that the effect of $\dot{\varepsilon}$ on chain elongation is about $10^{2}$ times as large as that of the shear rate. ${ }^{1}$

Figure 3 shows the typical $\dot{\varepsilon}$ dependence of crystallization behavior as a function of crystallization time $(t)$ observed using POM. We directly observed the so-called induction time $(\tau)$ defined as the onset of crystallization by means of POM. ${ }^{12}$ The $T_{\mathrm{c}}$ value was as high as $150^{\circ} \mathrm{C}$.

For crystallization under a quiescent field $\left(\dot{\varepsilon}=0 \mathrm{~s}^{-1}\right)$, nuclei nucleated at about $40 \mathrm{~min}$ and crystallization was completed after $21 \mathrm{~h}$ (Figure 3a). Therefore, we determined $\tau=40 \mathrm{~min}$ for $\dot{\varepsilon}=0$.

For crystallization at $\dot{\varepsilon}<60 \mathrm{~s}^{-1}$, nuclei nucleated at $\tau \cong 10 \mathrm{~min}$ and crystallization (meaning formation of spherulites) was complete after $11 \mathrm{~h}$. Figure $3 \mathrm{~b}$ shows a typical example of $\dot{\varepsilon}(S)=3 \mathrm{~s}^{-1}$. Therefore, crystallization was a little accelerated under weak elongational field.

When $\dot{\varepsilon}$ became larger than $60 \mathrm{~s}^{-1}$, a small fraction of NOCs crystallized and small spherulites formed gradually. Figure $3 \mathrm{c}$ shows a typical example of $\dot{\varepsilon}(S)=70 \mathrm{~s}^{-1}$. Although NOC formation was confirmed by retardation, it was difficult to obtain the $\tau$-value of NOCs because of very weak retardation in the early stage. Solidification was completed in about $2.5 \mathrm{~h}$.

When $\dot{\varepsilon} \geqslant 1 \times 10^{2} \mathrm{~s}^{-1}$, the NOC fraction significantly increased and the spherulite fraction decreased with increasing $\dot{\varepsilon}$. The NOCs crystallized within $1 \mathrm{~ms}$. Therefore, this crystallization was characterized by two $\tau$-values: $\tau \cong 1 \mathrm{~ms}$ for NOCs and $\tau \cong 8 \mathrm{~min}$ for spherulites.

For $\dot{\varepsilon}(\mathrm{L}) \geqslant 3 \times 10^{2} \mathrm{~s}^{-1}$, only NOCs were formed within $1 \mathrm{~ms}$ and NOC formation completed after $4 \mathrm{~ms}$. POM did not indicate any spherulites in the sample. Figure 3d shows a typical example for $\dot{\varepsilon}(\mathrm{L})=$ $1.3 \times 10^{3} \mathrm{~s}^{-1}$. Therefore, we concluded that the $\tau$ of NOC for $\dot{\varepsilon}(\mathrm{L})$ is less than $1 \mathrm{~ms}$,

$$
\tau \leq 1 \mathrm{~ms}
$$

because the time resolution of the high-speed camera used in this study was $1 \mathrm{~ms}$.

As the elongation took about $10 \mathrm{~ms}$, these results demonstrate that NOC crystallization was completed during elongation for $\dot{\varepsilon}(\mathrm{L})$. This result is in contrast to the crystallization of spherulites, which occurred slowly after cessation of elongation.

Figure 4 shows a plot of $\tau$ against $\dot{\varepsilon}$. $\tau$ was about $40 \mathrm{~min}$ for the quiescent condition. For comparison, a plot of $\dot{\varepsilon}=0 \mathrm{~s}^{-1}$ (quiescent) was superimposed on the left side of Figure 4 . With the increase in $\dot{\varepsilon}, \tau$ decreased slightly to $10^{3} \mathrm{~s}$ for $\dot{\varepsilon}(\mathrm{S})$. Over the entire range of $\dot{\varepsilon}(\mathrm{S}), \tau$ was almost constant against $\dot{\varepsilon}$ because the variation of $\tau$ was usually large. The $\tau$-values for spherulites and NOCs overlapped at $\dot{\varepsilon}<\dot{\varepsilon}^{*}$. For $\dot{\varepsilon}(\mathrm{L}) \geqslant \dot{\varepsilon}^{*}, \tau$ decreased discontinuously to $1 \mathrm{~ms}$, by as much as a factor of $10^{-6}$. The significant change in $\tau$ is one piece of evidence for the change in crystallization mechanism from heterogeneous to homogeneous nucleation, as shown later. 


\section{$\chi_{\mathrm{c}}$ of the NOC}

$\chi_{\mathrm{c}}$ for $\dot{\varepsilon}(\mathrm{L})$ was obtained by measuring the $\rho$ of the sample. As the $\rho$ of a typical sample was $\rho=M / V=0.94 \mathrm{~g} \mathrm{~cm}^{-3}, \chi_{\mathrm{c}}$ was obtained by ${ }^{13}$

$$
\chi_{c}=\frac{\rho_{c}}{\rho}\left(\frac{\rho-\rho_{a}}{\rho_{c}-\rho_{a}}\right)=0.92 \pm 0.02 \quad \text { for } \dot{\varepsilon}(L)
$$

where $\rho_{\mathrm{c}}=0.946 \mathrm{~g} \mathrm{~cm}^{-3}$ and $\rho_{\mathrm{a}}=0.852 \mathrm{~g} \mathrm{~cm}^{-3}$ are the densities of $\alpha_{2}$ form crystals (as will be shown later) and amorphous iPP at $25^{\circ} \mathrm{C}$, respectively. ${ }^{8,14} \chi_{\mathrm{c}}=0.92$ is the highest obtainable crystallinity for a bulky iPP solid, and normal iPP solid shows $\chi_{c}<0.7 .^{6}$

\section{$\dot{\varepsilon}$ dependence of the SAXS pattern and verification of NOC formation}

SAXS pattern of $\dot{\varepsilon}(L)$. Figures 5a-c show SAXS patterns of through-, edge- and end-views for $\dot{\varepsilon}(\mathrm{L})$, respectively. Through and edge patterns for $\dot{\varepsilon}(\mathrm{L})$ showed an 'oriented two-point pattern' along the MD, shown by arrow $A$. The end pattern did diffuse scattering pattern. Figure 6a shows a typical scattering intensity $\left(I_{\mathrm{X}}\right)$ profile for the through direction. The $I_{\mathrm{X}}$ was obtained by integration of the scattering intensity around the circular ring. The $I_{\mathrm{X}}$ showed only one dominant peak when plotted against the scattering vector $(q)$, with a two-point

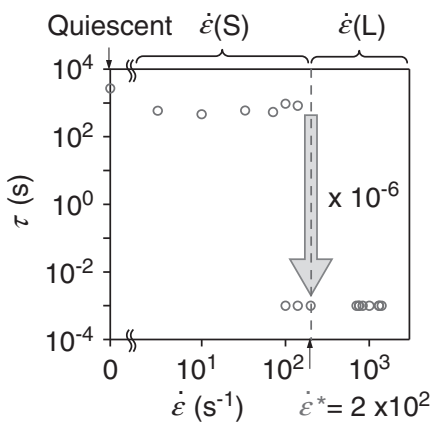

Figure 4 Plot of $\tau$ against $\dot{\varepsilon}$ including crystallizations by 1D and 2D compression. The discontinuous decrease of $\tau$ indicates the existence of $\dot{\varepsilon}^{*}$ and a change in the nucleation mechanism. pattern and a very weak peak at around $q=0.6 \mathrm{~nm}^{-1}$. The two-point pattern for the through and edge directions can be interpreted by the fact that the first peak of the diffraction pattern of so-called 1D paracrystals was dominant. ${ }^{15,16}$ This finding indicates that paracrystals have a low degree of order, with roughly the same degree of order as the 1D liquid-like packing of particles. ${ }^{17}$

As $\chi_{c} \cong 1$, the particle should be a single crystal-like crystallite, a component of an NOC. Note that the crystal size $(d)$ can be approximated by the distance between the nearest neighbors for $\chi_{\mathrm{c}} \cong 1$. As we obtained $q$ at the peak $q_{\mathrm{d}}=0.24 \mathrm{~nm}^{-1}, d$ was obtained by

$$
d \cong 2 \pi / q_{\mathrm{d}}=26 \mathrm{~nm} \text { for } \dot{\varepsilon}(\mathrm{L}) .
$$

As the two-point pattern showed a rather broad peak, $d$ values should show a large fluctuation, that is, the obtained $d \cong 26 \mathrm{~nm}$ should be the mean of a wide distribution. Steric observation and analysis of $d$ clarify that the crystal shape was roughly spherical. This proves that the above crystal is a nano-crystal with a size on the order of $\mathrm{nm}$ and that NOCs are composed of such crystals.

The very weak peak at $q \cong 0.6 \mathrm{~nm}^{-1}$ may be the second peak of the two-point pattern. A slightly larger $q$ of the second peak than the expected $q$ from the two-point pattern may be due to the polydispersity of the size of NOCs.

The trough pattern for $\dot{\varepsilon}(\mathrm{L})$ showed a 'streak' along the equatorial line, which indicates an isolated rod- or string-like structure along the MD according to the X-ray theory. ${ }^{17}$ An apparent streak along the equatorial line of the edge pattern comes from the total reflection of $\mathrm{X}$-ray, because the sample thickness was smaller than the size of the incident beam. On the basis of the results of SAXS, WAXS and TEM, we illustrate the structure of NOCs in Figure 6b. WAXS and TEM results will be shown later. In the illustrated model, nano-crystals with a mean size of $26 \mathrm{~nm}$ (shown in pink) are linked and oriented along the MD. As significant coherence (with respect to direction and distance) is observed between neighboring nano-crystals along the $\mathrm{MD}$, as analyzed above, the train of nano-crystals can be regarded as a rod or a string along the MD. As the coherence between nano-crystals in neighboring rods or strings is very weak, the rod or string can be regarded as an isolated rod.

The end-view represents a Fourier transform of the projection along/from the $c$ axis of the rod or string to the equatorial plane.

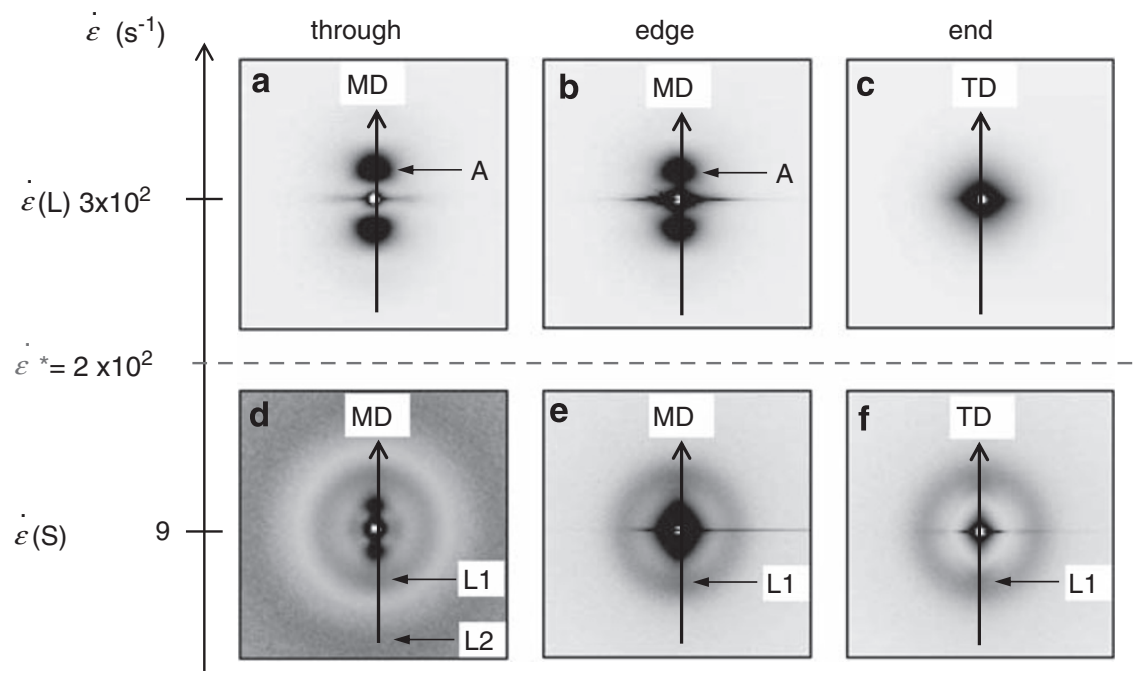

Figure $5 \dot{\varepsilon}$ dependence of SAXS patterns. Samples were crystallized by 1D compression. (a-c) $\dot{\varepsilon}(\mathrm{L})=3 \times 10^{2} \mathrm{~s}^{-1}$. (d-f) $\dot{\varepsilon}(\mathrm{S})=9 \mathrm{~s}^{-1}$. (a, d) Through-view. (b, e) Edge-view. (c, f) End-view. (d) The intensity of L2 is increased by a factor of 10 to show a weak ring. 

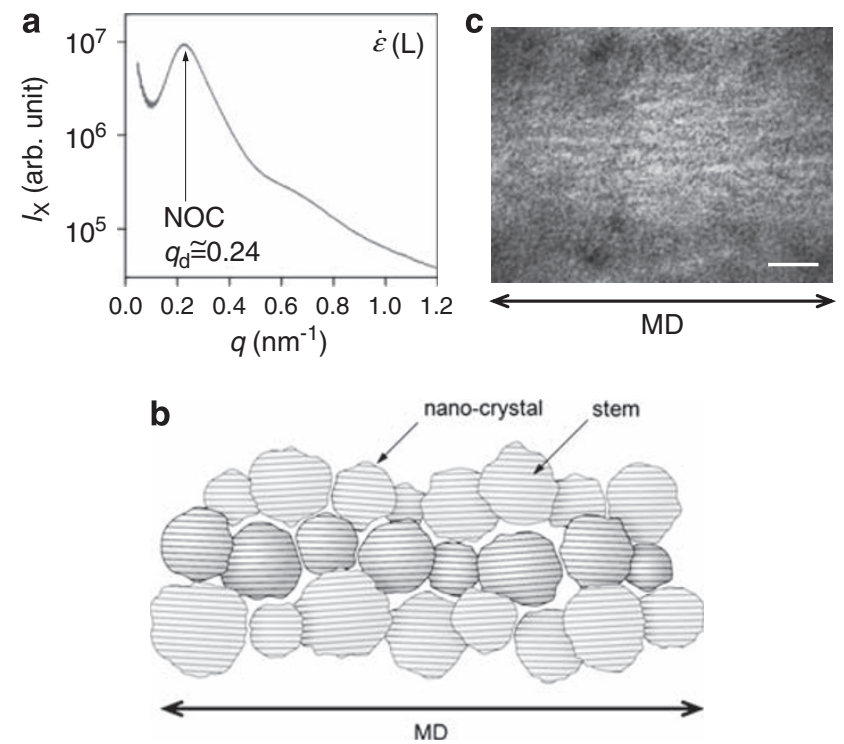

Figure $6 \dot{\varepsilon}$ dependence of SAXS $/ x$ and verification of NOC formation by TEM. (a) $/ x$ of the through-view against $q$ for $\dot{\varepsilon}(\mathrm{L})=3 \times 10^{2} \mathrm{~s}^{-1}$. Ix was obtained by all-around integration along a circular ring. The strong peak indicates the formation of NOC. Crystallized by 1D compression. (b) Schematic illustration of the structure of NOC. Connections between nano-crystals are omitted. (c) Edge-view transmission electron micrograph of NOC. $\dot{\varepsilon}(\mathrm{L})=1.25 \times 10^{3} \mathrm{~s}^{-1}$. Scale bar is $100 \mathrm{~nm}$. Crystallized by $2 \mathrm{D}$ compression. White dots confirmed the formation of nano-crystals.

As the electron density of nano-crystals on the projected plane was smeared and became uniform, we could only observe the shape factor of nano-crystals but not any unoriented peak such as the DebyeScherrer ring in Figure 5c.

Figure $6 \mathrm{c}$ shows a TEM of the sample for $\dot{\varepsilon}(\mathrm{L})$. The average size of the crystallite was about $26 \mathrm{~nm}$, and the crystals were oriented along the MD. Therefore, these results confirmed the presence of NOCs observed by SAXS.

Structures with a linear orientation in an X-ray field are commonly referred to as 'fiber structures'. ${ }^{15}$ Conventional fiber structures includes linearly oriented stacked lamellar systems and fringed micelles. As NOCs are linearly oriented nano-crystals but are different from both oriented stacked lamellar systems and fringed micelles, NOCs can be regarded as a new kind of fiber structure.

As Figures 2e and 3 show, the chains were oriented along the radial axis in the case of elongational crystallization with the $2 \mathrm{D}$ compression method, and chains were only elongated along the radial axis but not along the TD to the radial axis. This finding indicates that the highly nonequilibrium melt flow was only significantly generated along the radial direction above $\dot{\varepsilon}^{*}$. Therefore, the melt flow cannot exhibit equal biaxial stretching, but rather 1D stretching along the radial direction. The well-known equal biaxial stretching should only be expected for the slow $2 \mathrm{D}$ compression from a rod to a disk, that is, for small $\dot{\varepsilon}$, in which spherulites are usually generated.

SAXS patterns for $\dot{\varepsilon}(S)$ and quiescent conditions. Figures $5 \mathrm{~d}$, e and $\mathrm{f}$ show the SAXS patterns of through-, edge- and end-views for $\dot{\varepsilon}(\mathrm{S})$, respectively. These are mainly unoriented and weak two-point patterns. The typical $I_{\mathrm{X}}$ for the through direction presented in Figure 7, which was obtained by circular integration, showed a wellknown long-period pattern (L1 and L2). Therefore, the dominant

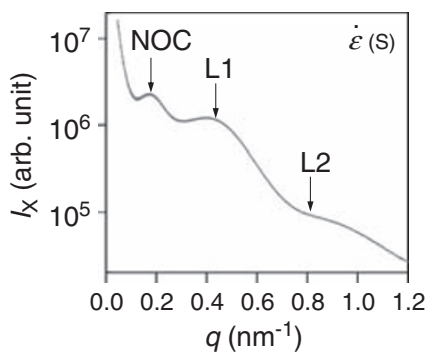

Figure 7 lx of the through-view against $q$ for $\dot{\varepsilon}(S)=9 s^{-1}$. Ix was obtained by all-around integration along the circular ring. L1 and L2 correspond to the well-known long period due to stacked lamellae in spherulites. Crystallized by $1 \mathrm{D}$ compression.

structure of $\dot{\varepsilon}(\mathrm{S})$ is a stacked lamellar structure. We obtained the long period $(L)$ from L1,

$$
L=2 \pi / q_{\mathrm{L} 1} \cong 15 \mathrm{~nm} \text { for } \dot{\varepsilon}(\mathrm{S})
$$

where $q_{\mathrm{L} 1}$ is $q$ at the peak of L1. The weak two-point pattern indicates that only tiny amounts of nano-crystals are mixed at the early stage of crystallization.

The SAXS pattern for the quiescent condition showed a well-known unoriented pattern over a long period, indicating the usual stacked lamellar structure. The observed $L$ for the quiescent condition was $L \cong 17 \mathrm{~nm}$ and $L \cong 25 \mathrm{~nm}$ for the $1 \mathrm{D}$ and $2 \mathrm{D}$ crystallization apparatuses, respectively. The latter is similar to the value for samples crystallized under isothermal conditions. ${ }^{9}$ The $1 \mathrm{D}$ case has a smaller $L$ because of the effect of the significant melt flow in an extruder die.

\section{$\dot{\varepsilon}$ dependence of the WAXS pattern}

WAXS pattern of $\dot{\varepsilon}(L)$. WAXS patterns of through-, edge- and endviews for $\dot{\varepsilon}(\mathrm{L})$ are shown in Figures $8 \mathrm{a}-\mathrm{c}$, respectively. Through and edge patterns showed a highly oriented 'fiber pattern' along the MD, indicating that molecular chains ( $c$ axes) were highly oriented along the MD. This pattern represents evidence of 'oriented' crystals, corresponding to the SAXS result for $\dot{\varepsilon}(\mathrm{L})$. As nano-crystals and $c$-axes were oriented along the $\mathrm{MD}$, we named this morphology the NOC. The end pattern showed an unoriented pattern, confirming that the NOC has a typical fiber structure.

Through and edge patterns also showed strong reflections of $\overline{2} 31$ and $\overline{1} 61$ of the $\alpha_{2}$ form, as shown by the arrows in Figures $8 \mathrm{a}$ and $\mathrm{b}$. As the $\overline{2} 31$ and $\overline{1} 61$ are characteristic reflections of the $\alpha_{2}$ form, the unit cell structure for $\dot{\varepsilon}(\mathrm{L})$ mainly belongs to the ordered form of the $\alpha_{2}$ form. ${ }^{8}$ The WAXS pattern also showed excess reflections near the meridian axis along the first layer line and between the 110 and 040 reflections of the $\alpha_{2}$ form. The former indicates the so-called $\mathrm{a}^{*}$-axis orientation of the crystals, and the latter indicates the 300 reflection of $\beta$-form crystals. ${ }^{6}$ As those crystals had a weak scattering intensity, they had little effect on physical properties. Therefore, this structural model of NOCs does not include $\mathrm{a}^{*}$-axis-oriented crystals or $\beta$-form crystals. $^{6}$

We obtained $f_{\mathrm{c}}$ by analyzing the 130 reflection for the through-view pattern (Figure 8a),

$$
f_{\mathrm{c}} \geqslant 0.9
$$

This clarified that molecular chains for $\dot{\varepsilon}(\mathrm{L})$ were highly oriented along the MD.

WAXS pattern of $\dot{\varepsilon}(S)$. In the case of $\dot{\varepsilon}(S)$, through-, edge- and endpatterns showed an unoriented Debye-Scherrer ring (Figures $8 \mathrm{~d}-\mathrm{f}$ ) 


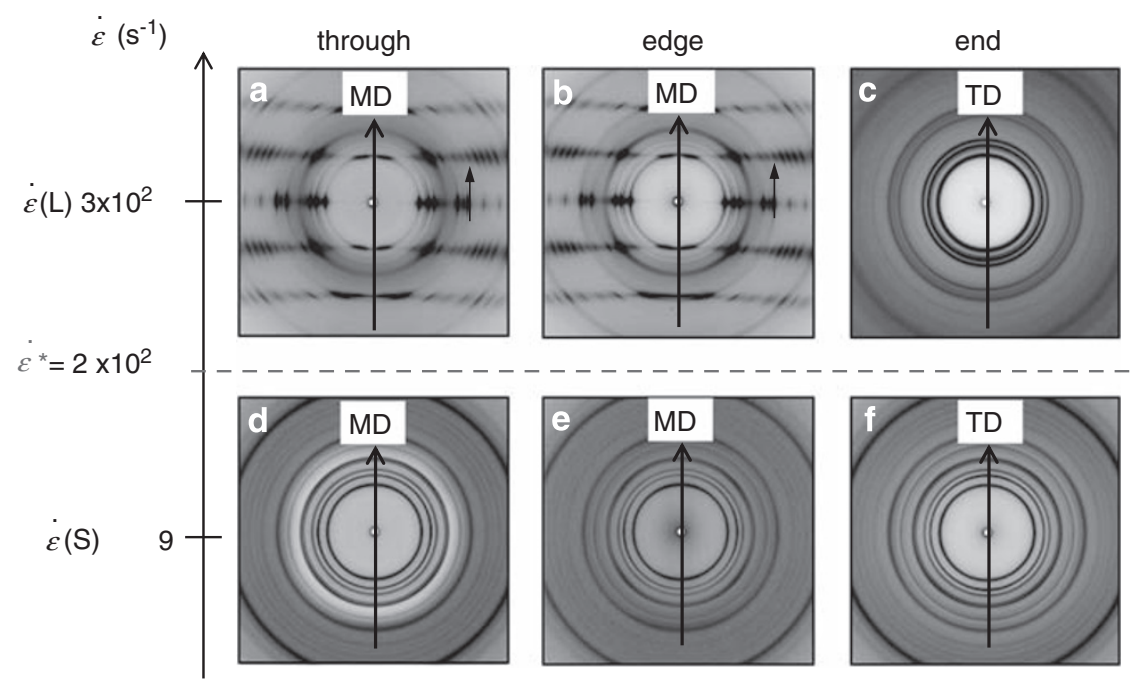

Figure $8 \dot{\varepsilon}$ dependence of WAXS patterns. Crystallized by 1D compression. (a-c) $\dot{\varepsilon}(\mathrm{L})=3 \times 10^{2} \mathrm{~s}^{-1}$. (d-f) $\dot{\varepsilon}(\mathrm{S})=9 \mathrm{~s}^{-1}$. (a, d) Through-view. (b, e) Edge-view. (c, f) End-view. (c-f) The intensity for $q>16 \mathrm{~nm}^{-1}$ is increased by a factor of 10 to show relatively weak reflections at large $q$. (a-c) and (d-f) confirmed typical fiber and Debye-Scherrer patterns, respectively.

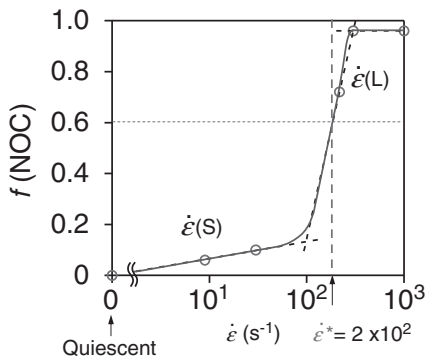

Figure 9 f(NOC) against $\dot{\varepsilon}$ including crystallizations by $1 \mathrm{D}$ and 2D compression. These results confirm a discontinuous increase at $\dot{\varepsilon}>1 \times 10^{2} \mathrm{~s}^{-1}$ and indicate $\dot{\varepsilon}^{*}=2 \times 10^{2} \mathrm{~s}^{-1}$.

and the unit cell structure belonged to the $\alpha_{1}$ form, as there were no $\overline{2} 31$ and $\overline{1} 61$ reflections.

\section{$\dot{\varepsilon}$ dependence of the NOC fraction $\mathrm{f}(\mathrm{NOC})$}

The NOC fraction $f(\mathrm{NOC})$ within the crystalline volume can be obtained from the analysis of WAXS intensity. $f(\mathrm{NOC})$ is defined by

$$
f(\mathrm{NOC}) \equiv \frac{I_{h k l}(\mathrm{NOC})}{I_{h k l}(\text { unori. })+I_{h k l}(\mathrm{NOC})},
$$

where $I_{h k l}(\mathrm{NOC})$ and $I_{h k l}$ (unori.) represent the total reflection intensity of an isolated $h k l$ reflection of the NOC and unoriented crystals such as spherulites, respectively. $I_{h k l}$ is defined by

$$
I_{h k l} \equiv \int I_{X}(\mathbf{q}) \mathrm{d} \mathbf{q}
$$

where $I_{\mathrm{X}}(\mathbf{q})$ is scattering intensity at $\mathbf{q}(h k l)$ and the integration was carried out within a region in the reciprocal space to which scattering from the $(h k l)$ reflection plane spread. The definition of this region depends on the scattering from the NOC or unoriented crystals.

As the WAXS patterns of the NOC and unoriented crystals show typical fiber and Debye-Scherrer patterns, respectively, these regions are well known in X-ray scattering theory. ${ }^{18}$ Therefore, integration can be easily carried out by analyzing the observed scattering patterns. When NOC and unoriented crystals are mixed together, the $I_{\mathrm{X}}(\mathbf{q})$ of
NOC can be estimated by subtracting the $I_{\mathrm{X}}(\mathbf{q})$ of unoriented crystals from the observed $I_{\mathrm{X}}(\mathbf{q})$.

Here, we obtained $f(\mathrm{NOC})$ by analyzing the 130 reflection for the through-view pattern (Figure 9). It is obvious that $f(\mathrm{NOC})=0$ in a quiescent field. With an increase in $\dot{\varepsilon}$ up to $1 \times 10^{2} \mathrm{~s}^{-1}, f(\mathrm{NOC})$ appeared and increased slightly to about 0.1 . For $\dot{\varepsilon} \geqslant 1 \times 10^{2} \mathrm{~s}^{-1}$, $f(\mathrm{NOC})$ started increasing significantly and saturated to

$$
f(\mathrm{NOC}) \cong 1 \text { for } \dot{\varepsilon} \geqslant 3 \times 10^{2} \mathrm{~s}^{-1}
$$

Therefore, we defined $\dot{\varepsilon}^{\star}$ as $\dot{\varepsilon}$, where $f($ NOC) becomes 0.6. From Figure 9, we obtained

$$
\dot{\varepsilon}^{*}=2 \times 10^{2} \mathrm{~s}^{-1}
$$

\section{Nucleation mechanism of the NOC}

$\dot{\varepsilon}$ dependence of size and crystal number density. Figure 10 plots $d$ against $\dot{\varepsilon}$ after completion of solidification. The $d$ values for quiescent and $\dot{\varepsilon}(\mathrm{S})$ conditions were the diameter of spherulite obtained by POM and that for $\dot{\varepsilon}(\mathrm{L})$ was the nano-crystal size obtained by SAXS. This figure clarifies that with increasing $\dot{\varepsilon}, d$ decreased slightly for $\dot{\varepsilon}(\mathrm{S})$ and decreased discontinuously by as much as a factor of $10^{-3}$ for $\dot{\varepsilon}(\mathrm{L})$.

We obtained the number density $(v)$ of spherulites and nanocrystals against $\dot{\varepsilon}$ (Figure 10) using the approximate equation,

$$
v \cong d^{-3}
$$

With increasing $\dot{\varepsilon}, v$ increased slightly for $\dot{\varepsilon}(\mathrm{S})$ and increased discontinuously by as much as a factor of $10^{9}$ for $\dot{\varepsilon}(\mathrm{L})$.

Because a spherulite is formed from a nucleus, the small $v$ of the spherulite indicates that the number density of the nucleus should be small for quiescent and $\dot{\varepsilon}(S)$ conditions. On the other hand, the significant increase in $v$ for $\dot{\varepsilon}(\mathrm{L})$ indicates that the number density of the nucleus should increase for $\dot{\varepsilon} \geqslant \dot{\varepsilon}^{*}$.

Relationship between $v$ and nucleation mechanism. We now present evidence that the discontinuous increase of $v$ is due to the change in nucleation mechanism at $\dot{\varepsilon}^{*}$ from 'heterogeneous nucleation' to 'homogeneous nucleation'.

Heterogeneous nucleation is a process in which the nucleus nucleates on a surface at the site of an impurity or heterogeneity 


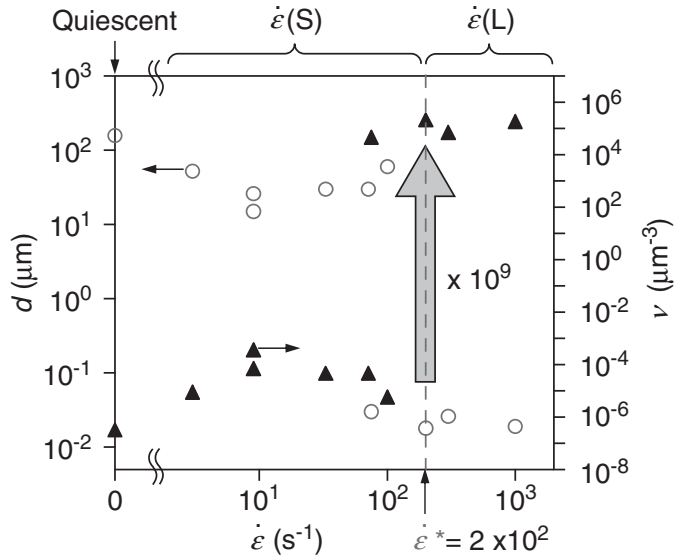

Figure $10 d(O)$ and $v(\mathbf{\Lambda})$ against $\dot{\varepsilon}$, including crystallizations by $1 \mathrm{D}$ and 2D compression. $d$ and $v$ correspond to the left and right axes, respectively.

that functions as a 'nucleating agent' because of attractive interactions at the interface between the heterogeneity and the nucleus. ${ }^{19}$ Homogeneous nucleation is a process in which the nucleus nucleates without any help from an impurity or heterogeneity. ${ }^{19}$ Only a small amount of heterogeneity is essentially included in the melt in nature, and most nucleation from the bulky melt occurs by heterogeneous nucleation. It is more difficult for homogeneous nucleation to occur than heterogeneous nucleation because of the much higher free-energy activation barrier for the formation of a critical nucleus near the melting temperature $\left(T_{\mathrm{m}}\right) \cdot{ }^{19}$

The significant increase in $v$ for $\dot{\varepsilon} \geqslant \dot{\varepsilon}^{*}$ in this study cannot be attributed to the increase in the number density of heterogeneity, as it is logically impossible that the number of heterogeneities increases discontinuously by $10^{9}$ times only when $\dot{\varepsilon}$ becomes larger than $\dot{\varepsilon}^{*}$. Therefore, the increase in $v$ is evidence of the change from heterogeneous to homogeneous nucleation. Thus far, homogeneous nucleation near $T_{\mathrm{m}}$ has been limited to droplet melt. ${ }^{20}$ Therefore, this finding is the first to show homogeneous nucleation occurring from the bulky melt for any material near $T_{\mathrm{m}}$, which results in NOC formation and a significant improvement in properties as shown later.

As there is no concrete evidence on homogeneous nucleation even for that 'orientation- or stress-induced crystallization occurs during the melt processing of polymers' in any studies in literature, our result is the first to confirm homogeneous nucleation from the bulky melt. We showed concrete evidence of homogeneous nucleation by showing a significant increase in $v$ by a factor of $10^{9}$ during nucleation of NOCs, as large as that during crystallization of conventional spherulites.

Nucleation mechanism under elongational flow. We verified the mechanism of NOC nucleation: Figure 11a illustrates homogeneous nucleation by compression. We focus on one Gaussian chain within the bulky melt. ${ }^{21}$ Gaussian chains should be elongated along the MD, that is, the elongational direction, when the bulky melt is being compressed at the condition of $\dot{\varepsilon}(\mathrm{L}) \geqslant \dot{\varepsilon}^{*}$. At $\dot{\varepsilon}(\mathrm{L}) \geqslant \dot{\varepsilon}^{*}$, the elongational force overcomes the entropic relaxation of randomization. At the same time, the chains are locally packed in parallel. Because the strong elongation can lead to the formation of many parallel packed chains, many of the melted polymer chains are oriented along the elongational direction. Thus, the bulky melt becomes similar to a liquid crystal, fitting the concept of the 'oriented-melt'. It is natural to imagine that

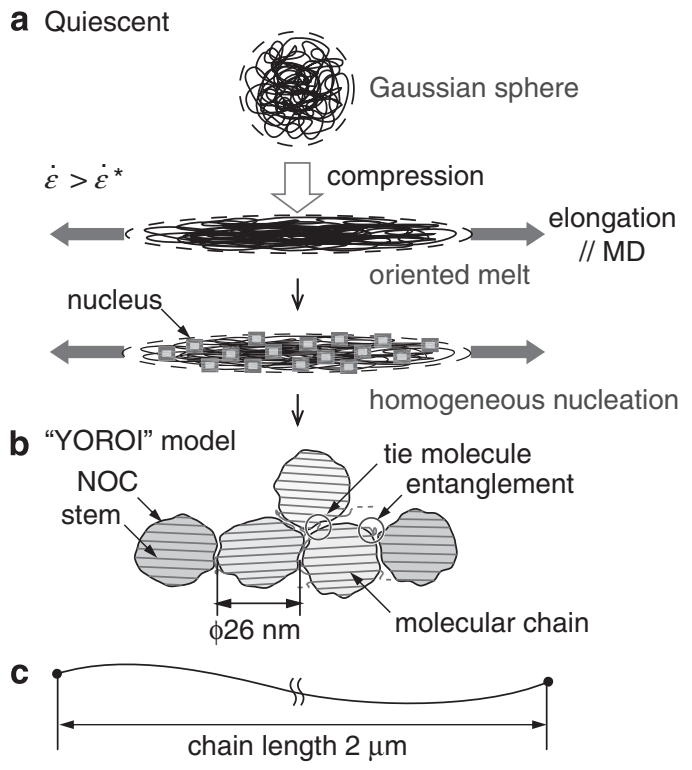

Figure 11 Schematic illustration of the NOC nucleation mechanism. (a) A Gaussian sphere within the isotropic melt under a quiescent field. Compression at $\dot{\varepsilon}>\dot{\varepsilon}^{*}$ induces formation of an 'oriented melt' by significant chain elongation, which induces homogeneous nucleation. (b) Schematic illustration of the 'YOROI model' of the NOC structure. (c) Schematic illustration of one molecular chain virtually extended to its full length.

the nucleus nucleates easily within the parallel packed chains. An infinite number of nuclei are generated by homogeneous nucleation, and they grow instantaneously into nano-crystals before the melt structure randomizes because of entropic relaxation (Figure 11b).

Logically speaking, the definition of $\dot{\varepsilon}^{*}$ is a critical $\dot{\varepsilon}$, in which the $\tau$ of the homogeneous nucleation becomes shorter than the lifetime of the oriented melt $\left(\tau_{\mathrm{m}}\right)$. The nucleation significantly depends on the degree of supercooling $(\Delta T)$, which is defined by the difference between $T_{\mathrm{m}}{ }^{0}$ and $T_{\mathrm{c}} \cdot \tau_{\mathrm{m}}$ represents the entropic relaxation time from the oriented melt to the isotropic melt. $\tau_{\mathrm{m}}$ depends on $M_{\mathrm{w}}$ and on the distribution of molecular weight $(M)$. Therefore, $\dot{\varepsilon}^{*}$ would depend on $\Delta T$ and $M_{\mathrm{w}}$. It is important to control the morphology and structure by changing $\Delta T, M_{\mathrm{w}}$ and the $M$ distribution. $\dot{\varepsilon}^{*}$ is a discontinuous point at which the morphology, structure and physical properties (shown later) discontinuously change.

For $\dot{\varepsilon}(\mathrm{S})<\dot{\varepsilon}^{*}$, although weak parallel packing of chains is induced by elongation, most of the parallel packing will be relaxed before nucleation by entropic relaxation. Therefore, it was impossible to reach a sufficiently high number density of nano-crystals under this condition.

The 'YOROI' model of the NOC. During growth, molecular entanglements originally included within the melt should be condensed to the interface between the nano-crystals. Within one nano-crystal, $10^{3}$ 'stems' of molecular chains are packed in parallel. One chain should three-dimensionally interpenetrate and connect the nano-crystals more than $10^{2}$ times, as the mean extended length of one molecular chain is about $2 \mu \mathrm{m}$ (Figure 11c). Note that one molecule should take on an elongated 'random walk' conformation even after experiencing critical elongation. This means that the parallel packing should be locally realized and that the overall conformation should be an elongated random walk. Therefore, nano-crystals are tightly connected three dimensionally by carbon-carbon covalent bonds named 'tie 
molecules' and entanglements. We name the schematic illustration of NOCs as the 'YOROI' model because the structure is similar to the armor of samurai in ancient Japan.

From the $\chi_{\mathrm{c}}$, we estimated the mean thickness of the interface between nano-crystals to be as narrow as $0.5 \mathrm{~nm}$. As a strong SAXS intensity of the two-point pattern of NOC was observed, there should be a significant difference in electron density between the interior of the nano-crystal and the interface. It is reasonable for the electron density of the interface to be less than that of the nano-crystal because the entanglement requires much more free volume than molecular packing within a crystal.

\section{Physical properties of the NOC}

Tensile stress. Figure 12a presents typical stress $(\sigma)$-strain $(\varepsilon)$ curves for $\dot{\varepsilon}(\mathrm{L})$ and $\dot{\varepsilon}(\mathrm{S})$. Tensile stress at break $\left(\sigma_{\mathrm{B}}\right)$ was $\sigma_{\mathrm{B}}=2.1 \times 10^{2} \mathrm{MPa}$ for $\dot{\varepsilon}(\mathrm{L})$. This $\sigma_{\mathrm{B}}$ for $\dot{\varepsilon}(\mathrm{L})$ was about 7 times as large as that for conventional sheets of $\mathrm{iPP}$ and high-density polyethylene $\left(\sigma_{\mathrm{B}} \cong 30 \mathrm{MPa}\right){ }^{22} \sigma_{\mathrm{B}}$ for $\dot{\varepsilon}(\mathrm{S})$ was $\sigma_{\mathrm{B}}=32 \mathrm{MPa}$, comparable to that for iPP and high-density polyethylene. We also compared $\sigma_{\mathrm{B}}$ for $\dot{\varepsilon}(\mathrm{L})$ with other bulky polymer samples thicker than $0.1 \mathrm{~mm}$; for example, $\sigma_{\mathrm{B}} \cong 45 \mathrm{MPa}$ for polyethylene terephthalate, $\sigma_{\mathrm{B}} \cong 50 \mathrm{MPa}$ for polystyrene and $\sigma_{\mathrm{B}} \cong 85 \mathrm{MPa}$ for nylon66. ${ }^{22}$ Thus, $\sigma_{\mathrm{B}}$ for $\dot{\varepsilon}(\mathrm{L})$ was the highest within conventional polymeric materials and was twice as large as that of aluminum and comparable to that of duralumin. ${ }^{23}$

Specific tensile strength. We obtained the specific tensile strength defined by $\sigma_{\mathrm{s}} \equiv \sigma_{\mathrm{B}} / \rho_{\mathrm{s}}$, where $\rho_{\mathrm{s}}$ is specific gravity. $\sigma_{\mathrm{s}}$ is a useful index for comparison with different materials. $\sigma_{\mathrm{s}}=2.2 \times 10^{2} \mathrm{MPa}$ for $\dot{\varepsilon}(\mathrm{L})$ was a few times larger than that of the steel used for car bodies, which has $\sigma_{\mathrm{s}} \cong(0.6-1.3) \times 10^{2} \mathrm{MPa}^{23}$ Therefore, the specific strength of NOCs is a few times higher than that of common steel with the same mass.

Young's modulus. Young's modulus $(E)$ of the sheet was $E=4.1 \mathrm{GPa}$ for $\dot{\varepsilon}(\mathrm{L})$. This is a high modulus, compared with $E=1.4 \mathrm{GPa}$ for $\dot{\varepsilon}(\mathrm{S})$, $E=1.5 \mathrm{GPa}$ for conventional iPP, $E=1.3 \mathrm{GPa}$ for high-density polyethylene, $E=2.3 \mathrm{GPa}$ for polyethylene terephthalate and $E=2.6 \mathrm{GPa}$ for polystyrene and nylon 66.22

$\sigma_{\mathrm{B}}$ and $E$ values of conventional sheets of polymers are usually much smaller than those of fiber. Therefore, mechanical properties of the NOC sheet were compared with those of conventional sheets in this study rather than with fibers.

Toughness. Toughness was semiquantitatively tested by repetitive folding of the sheet. The sample of $\dot{\varepsilon}(\mathrm{L})$ was not even cut after 100fold along the MD and the TD, and the fold-back lines in both directions remained transparent without becoming clouded, confirming that NOC show ultra-high toughness. The quiescent and $\dot{\varepsilon}(\mathrm{S})$ samples became clouded at the fold-back point because of generation of voids after only a few folds along the MD and the TD. Although they could not be cut easily, the fold-back points became very weak. In the range of $\dot{\varepsilon} \cong \dot{\varepsilon}^{*}$, the sample was easily cut when it was folded along MD only a few times, although it was tough along TD, which indicates that the sample of $\dot{\varepsilon} \cong \dot{\varepsilon}^{*}$ was brittle along MD. Therefore, it is important for industrial purposes to crystallize NOC at $\dot{\varepsilon} \geqslant \dot{\varepsilon}^{*}$ to ensure maximal toughness.

Thermal resistance. Figure $12 \mathrm{~b}$ presents typical thermal resistance tests for $\dot{\varepsilon}(\mathrm{L})$, plotting the normalized size $\left(l / l_{0}\right)$ against temperature $(T)$, where $l$ is lateral size and $l_{0}$ is initial lateral size at room temperature. Values of $l / l_{0}$ did not change up to $176^{\circ} \mathrm{C}$ and then $\left|l / l_{0}\right|$ suddenly changed more than $3 \%$. Therefore, a heatproof temperature $T_{\mathrm{h}}=176{ }^{\circ} \mathrm{C}$ was obtained for $\dot{\varepsilon}(\mathrm{L})$. We also observed the $T_{\mathrm{h}} \mathrm{S}$
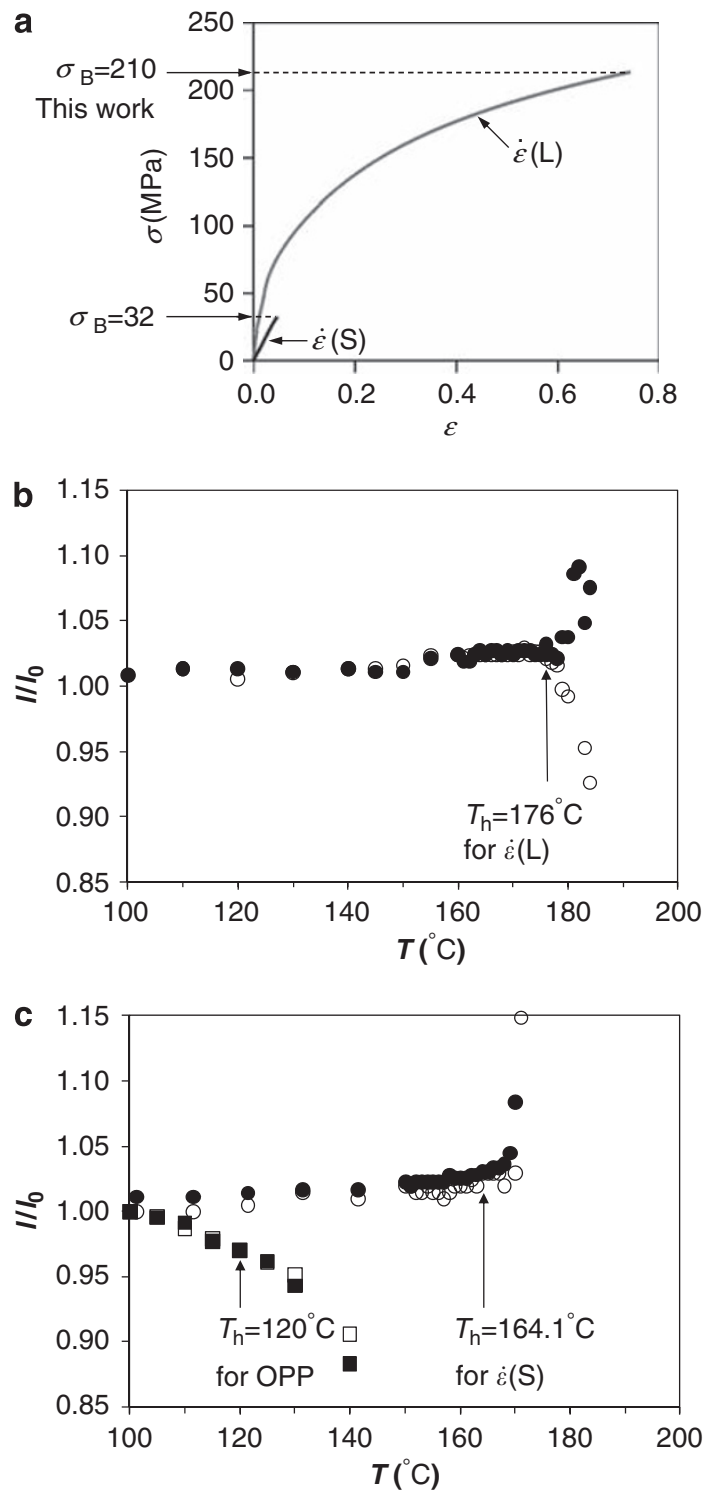

Figure 12 Physical properties. (a) Typical stress-strain curves for $\dot{\varepsilon}(\mathrm{L})=3 \times 10^{2} \mathrm{~s}^{-1}$ and $\dot{\varepsilon}(\mathrm{S})=9 \mathrm{~s}^{-1}$. Samples were crystallized by 1D compression. The $\sigma_{\mathrm{B}}$ for $\dot{\varepsilon}(\mathrm{L})$ is about 7 times as large as that for $\dot{\varepsilon}(\mathrm{S})$ and/or conventional iPP sheet. (b, c) Thermal resistance test from $/ / I_{0}$ against $T$. $\dot{\varepsilon}(\mathrm{L})$ and $\dot{\varepsilon}(\mathrm{S})$ were crystallized by $1 \mathrm{D}$ compression. (b) $\dot{\varepsilon}(\mathrm{L})=3 \times 10^{2} \mathrm{~s}^{-1}$. $\bigcirc$ and $\bullet$ indicate $I_{\mathrm{MD}}$ and $/_{\mathrm{TD}}$, respectively. A significantly high $T_{\mathrm{h}}=176^{\circ} \mathrm{C}$ was obtained. (c) $\dot{\varepsilon}(\mathrm{S})=9 \mathrm{~s}^{-1}$ and commercial OPP. $\bigcirc$ and $\bullet$ indicate $I_{\mathrm{MD}}$ and $l_{\text {TD }}$ for $\dot{\varepsilon}(\mathrm{S})$, respectively. $\square$ and $\boldsymbol{\square}$ indicate $I_{\mathrm{MD}}$ and $l_{\text {TD }}$ for OPP, respectively.

for $\dot{\varepsilon}(\mathrm{S})$ and for commercial biaxially oriented polypropylene (OPP) (Figure $12 \mathrm{c}$ ). $T_{\mathrm{h}}=164.1^{\circ} \mathrm{C}$ for $\dot{\varepsilon}(\mathrm{S})$ and $T_{\mathrm{h}} \cong 120^{\circ} \mathrm{C}$ for OPP. As the thermal resistance of OPP is known to be the highest among conventional polymers, it is concluded that the $T_{\mathrm{h}}$ of $\dot{\varepsilon}(\mathrm{L})$ was much higher than that of OPP, and this fact satisfies the condition requested for 'super engineering plastics', that is, $T_{\mathrm{h}}>150{ }^{\circ} \mathrm{C} .{ }^{24}$ Therefore, we anticipate broad usefulness of these materials in practical or industrial applications.

Transparency. We compared the transparency by 'haze' for various polymer sheets of $0.3 \mathrm{~mm}$ thickness. Haze for $\dot{\varepsilon}(\mathrm{L})$ was as small as $0.9 \%$, which was significantly smaller than that for well-known 
transparent plastics of polystyrene with haze $=1.9 \%$. The haze for $\dot{\varepsilon}(\mathrm{S})$ was $47 \%$. The hazes of the other typical samples were $6 \%$ for commercial overhead projector (polyethylene terephthalate) sheet, $10 \%$ for OPP and $25 \%$ for a commercial clear holder of iPP.

Discussion: origin of ultra-high performance for NOC. These results confirm that NOC show ultra-high performance for physical properties, indicating that nano-crystals of inorganic materials show high performance as predicted by Hall-Petch's law. ${ }^{25,26}$ The reason for the simultaneous realization of the large tensile strength and high toughness of the NOC is likely the three-dimensional connection of nanocrystals by tie molecules and entanglements as shown in the YOROI model. Therefore, the molecular connection in the NOC should correspond to dislocation in inorganic materials. The high heat resistance of $\dot{\varepsilon}(\mathrm{L})$ should be because of high crystallinity.

Therefore, this study of the crystallization mechanism of polymers from the melt under a strong external field and of the structure formation and properties of those crystals is interesting both scientifically and industrially. The NOCs developed here may be used in a broad array of fields such as livingware and industrial supplies, in which conventional polymers cannot currently be used. The NOCs will be able to replace metals or ceramics, thus reducing the weight of products, contributing to the development of a sustainable society.

\section{CONCLUSIONS}

We succeeded in elongational crystallization of iPP by a new method of 'compressing' the supercooled melt. When the elongational strain rate $(\dot{\varepsilon})$ became larger than the critical value $\dot{\varepsilon} \geqslant \dot{\varepsilon}^{*} \cong 2 \times 10^{2} \mathrm{~s}^{-1}$, the morphology discontinuously changed from well-known spherulite crystals to NOC. $\dot{\varepsilon}^{*}$ is defined as an $\dot{\varepsilon}$ at which the fraction of NOCs $f($ NOC) becomes larger than 0.6. The NOCs showed a high crystallinity as high as $\chi_{c}=0.92 \pm 0.02$. SAXS and WAXS patterns of NOCs showed an oriented two-point pattern and a highly oriented fiber pattern along the machine (or elongational) direction, respectively. The unit cell structure of the NOC belonged to the $\alpha_{2}$ form.

We obtained the number density $(v)$ of spherulites and nanocrystals by approximation $v \cong d^{-3}$. Here, $d$ is the size of the spherulite and/or the nano-crystal. When $\dot{\varepsilon}$ became larger than $\dot{\varepsilon}^{*}, v$ discontinuously increased by as much as a factor of $10^{9}$. Therefore, we concluded that the nucleation mechanism discontinuously changed from heterogeneous to homogeneous nucleation for $\dot{\varepsilon} \geqslant \dot{\varepsilon}^{*}$. On the basis of the above structural and crystallization studies, we propose the 'YOROI' model of NOCs.

NOCs showed ultra-high performance including a high tensile stress at break, $\sigma_{\mathrm{B}}=2.1 \times 10^{2} \mathrm{MPa}$, a high Young's modulus, $E=4.1 \mathrm{GPa}$, a high thermal resistance, $T_{\mathrm{h}}=176^{\circ} \mathrm{C}$, and a high transparency, haze $=0.9 \%$, for $0.3 \mathrm{~mm}$ sheet thickness.

\section{ACKNOWLEDGEMENTS}

Synchrotron radiation experiments were conducted at BL40B2 in SPring-8 with the approval of the Japan Synchrotron Radiation Research Institute (JASRI) (proposal no.2006A1304, 2006B1185, 2007A1567, 2007B1173, 2008B1611, 2009A1331 and 2009B1385). We thank H Kimura, Kawasaki Development Center, SunAllomer Ltd, for development and construction of the roll-type apparatus, K Yamada, Kawasaki Development Center, SunAllomer Ltd, for TEM observations and M Hikosaka and S Hikosaka, Hiroshima University, for experimental assistance.

1 Go, Y., Matsusawa, S. \& Yamaura, K. Ryudo niyoru Koubunnshi no Kessyouka 369-377 (Koubunshikankoukai, Kyoto, 1980).

2 Kotaka, T., Kojima, A. \& Okamoto, M. Elongational flow opto-rheometry for polymer melts. Rheologica Acta 36, 646-656 (1997).

3 Wunderlich, B. Macromolecular physics, vol.1, 348-357 (Academic press, New York and London, 1973).

4 Yamazaki, S., Watanabe, K., Okada, K., Yamada, K., Tagashira, K., Toda, A. \& Hikosaka, M. Formation mechanism of shish in the oriented melt (I) -bundle nucleus becomes to shish. Polymer 46, 1675-1684 (2005).

5 Hikosaka, M., Okada, K., Watanabe, K. \& Yamazaki, S. in Advances in Polymer Science, vol. 191, 137-186 (Springer-Verlag, Berlin, 2005).

6 Moore, E. P. Polypropylene handbook Ch. 3 (Kogyo Chosakai, Tokyo, 1998).

7 The Society of Rheology, Japan. Kohza, Rheology 9-13 (Koubunshikankoukai, Kyoto, 1992).

8 Hikosaka, M. \& Seto, T. The order of molecular chains in isotactic polypropylene crystals. Polymer J. 5, 111-127 (1973).

9 Yamada, K., Hikosaka, M., Toda, A., Yamazaki, S. \& Tagashira, K. Molecular weight dependence of equilibrium melting temperature and lamellar thickening of isotactic polypropylene with high tacticity. J. Macromol. Sci. Phys. B 42, 733-752 (2003).

10 Alexander, L. E. X-ray diffraction methods in polymer science Ch. 4 (Kagaku Dohjin, Kyoto, 1973).

11 Awaya, Y. Kobunnshi Sozai no Henko Kenbikyo Nyumon 1-255 (Agne Gijutsu Center, Tokyo, 2001).

12 Frisch, H. L. Time lag in nucleation. J. Chem. Phys. 27, 90-94 (1957).

13 Alexander, L. E. X-ray diffraction methods in polymer science Ch. 3 (Kagaku Dohjin, Kyoto, 1973).

14 Qirk, R. P. \& Alsamarriaie, M. A. A. Polymer Handbook V21-V28 (Awiley-interscience, New York, 1989).

15 Kakudo, M. \& Kasai, N. Koubunshi X-sen Kaisetsu Ch. 5 (Maruzen, Tokyo, 1968)

16 Kakudo, M. \& Kasai, N. Koubunshi X-sen Kaisetsu 295-307 (Maruzen, Tokyo, 1968).

17 Guinier, A. Theorie et technique de la radiocristallographie Ch. 10-11 (Dunod, Paris, 1964).

18 Nitta, I. X-sen kessyougaku, the first volume 117-121 (Maruzen, Tokyo, 1959) (in Japanese).

19 Price, F. P. in Nucleation (ed. Zettlemoyer, A. C.) Ch. 8 (Marcel Dekker, New York, 1969).

20 Cormia, R. L., Price, F. P. \& Turnbull, D. Kinetics of crystal nucleation in polyethylene. J. Chem. Phys. 37, 1333-1340 (1962).

21 Tanaka, F. Koubunshi no Butsurigaku 35-38 (Shokabo, Tokyo, 1994).

22 Kan-no, T. in Plastics processing databook (ed. The Japan Society for Technology of Plasticity) 39 (Nikkan Kogyo Shimbun, Tokyo, 2002).

23 lida, S. Butsuri Teisu-hyo 185-189 (Asakura Publishing Co., Ltd., Tokyo, 1976).

24 Kikutani, T. \& Takemura, K. in Plastic for processing (ed. The Japan Society of Polymer Processing) 6 (Kogyo Chosakai, Tokyo, 2006).

25 Eshelby, H. D., Frank, F. C. \& Nabarro, F. R. N. The equilibrium of linear arrays of dislocations. Phil. Mag. 42, 351-364 (1951).

26 Hall, E. O. The deformation and ageing of mild steel: III Discussion of results. Proc. Phys. Soc. B 64, 747-753 (1951). 